\title{
A COIMPARATIVE STUDY RELATING WATER DRINIKING TEST AND INTRA OCULAR PRESSURE IN DIFFERENT AGE GROUPS
}

KEY WORDS: Glaucoma Intraocular pressure, Water drinking.

\section{Dr. Thangerani} Raajaseharan

HOD, Ophthalmology, Government Stanley medical college, Chennai.

Dr. S.R.

Raajaseharan

Dr. Aishwarya

Thanasekaran

Professor, Physiology, KMCH Coimbatore.

Dr. Nihma Arif $\boldsymbol{A}$

$\mathbf{M}$ *

Junior Resident in Ophthalmology, Government Stanley medical college, Chennai. chennai. *Corresponding Author

AIM: To determine the behavior of IOP in different age groups among normal populations after water drinking test for a period of time.

MATERIALS AND METHODS: This is a prospective study involving normal healthy individual taken from different age groups 20-50 years, with no relevant past medical and surgical history. Patients were instructed to drink lLitre of water over a period of 5-10minutes after 8 hours of overnight fasting and the IOP was checked before drinking water and every 15 minutes for 1 hour period followed by 2 hours after drinking water using schiotz tonometer. The difference in baseline IOP and IOP at 1 hour and 2 hour was evaluated among different age groups .

RESULT: 100 patients from the age groups 20-50 years were studied.The mean baseline IOP was in the range of $12.4 \mathrm{mmHg}$ and the mean IOP after 1 hour was $15.3 \mathrm{mmHg}$.A difference of 4-4.5 mmHg were noted in the age group of 4050 years. The IOP was gradually decreasing to $12.78 \mathrm{mmHg}$ over a period of 2 hours afterWDT.

CONCLUSION: The higher age group the more the rise in the IOP after WDT which concludes that as the age increases drinking litres of water over a few minutes will substantially increase the IOP, which might damage the optic nerve.

\section{INTRODUCTION:}

Glaucoma is a group of progressive optic neuropathies characterized by the degeneration of retinal ganglion cells and of the retinal nerve fiber layer, resulting in morphological changes in the optic nerve head and specific pattern of visual field defect, where IOP can be a key modifiable factor .

IOP is determined by the aqueous humor formation and aqueous outflow and its resistance at the angle. Normal range of IOP is $10-21 \mathrm{mmHg}$.

\section{Risk factors for elevated IOP.}

- Old age

- Family History

- Race/Ethnicity

- Central corneal thickness (CCT)

- Low ocular perfusion pressure

- Diabetes mellitus-Type 2

- Myopia

Water drinking test: WDT is a provocative test/stress test which can be used in the diagnosis of primary open angle glaucoma and also a practical method of estimating peak diurnal IOP ${ }^{3}$.The increase in IOP during WDT have shown it to be a risk factor for the development and progression of glaucoma ${ }^{4,5}$.It indirectly measure the outflow facility of the anterior chamber angle. Smaller fluid challenge volumes would also more closely replicate the volumes of water consumed in everyday life, which reflects the change in IOP to small fluid volumes consumed in daily living activities. Every small rise in IOP is significant as every millimeter of mercury rise in IOP increases the risk of glaucoma progression ${ }^{6}$.

The purpose of this study is to know the changes in the outflow pathway with respect to increasing age which further influence the IOP and determines the susceptibility of further optic nerve damage.

\section{MATERIALS AND METHOD:}

This is a hospital based prospective study in an outpatient department where 100 normal patients from four age groups $20-30,30-40,40-50,50-60$ years with 25 patients from each age groups were selected.

Patients with any ocular infections, corneal diseases, previous history of glaucoma, with shallow anterior chamber on slit lamp examination, history of previous topical medications or ocular surgeries, history of any systemic diseases or systemic medications were excluded from the study.

All the patients were instructed to be in overnight fasting for a period of 8 hours prior to the test. Patients were instructed to drink lLitre of water over a period of 5-10 minutes. A baseline IOP was recorded prior to drinking water with a schiotz tonometer using all the 3 weights( $5.5 \mathrm{gms}, 7.5 \mathrm{gms}, 10 \mathrm{gms})$ and the average was taken. Subsequently the IOP was measured by the same method for every 15 mins for 1 hour after drinking water. The IOP after 2 hours of drinking water was also recorded.

The difference between the baseline IOP and the IOP after 1 hour was compared in each age group. The difference shows a gradual increase as the age increases. A maximum of 4-4.5 mmHg was seen among higher age groups.

The IOP after 2 hours of water drinking shows a gradual decline towards the baseline.

\section{RESULTS:}

Among 100 patients, after drinking 1 Litre of water IOP shows a gradual increase after 15 minutes onwards.

The mean baseline IOP in the ascending age groups was in the range of $11.27,11.42,13.20,13.75 \mathrm{mmHg}$ and the mean IOP after 1 hour of WDT was 13.46,14.26,16.14,17.50mmHg in the ascending age groups. As age increases the difference between baseline IOP and the IOP after 1 hour period also increases. A rise of about $3.5-4 \mathrm{mmHg}$ was noted in the age group of 50-60 years. In all age groups the IOP came to baseline by 2 hours ofWDT. 


\section{DISCUSSION:}

It is well recognized that IOP is a modifiable risk factor ${ }^{8,9}$. its essential to know the factors influencing the IOP variations in activities of daily living. IOP being a detrimental factor, leading to the glaucomatous optic nerve damage implies the "target pressure" varies among different individuals. IOP within the normal range need not always be the target pressure as in case of 'normal tension glaucoma'(NTG), where IOP is within the normal range (less than $21 \mathrm{mmHg}$ ) can even lead to an optic nerve damage and visual field defect. The physiological changes in the aqueous outflow with advancing age could manifest as NTG.

As the age advances the extracellular matrix (ECM) in the cribriform portion of trabecular meshwork(TM), beneath the schlemm's canal and the ciliary muscles also increases and the trabecular lamellae thickness is increased. These areas are less perfused due to higher resistance ${ }^{1}$.

Transforming growth factor-beta2(TGF-B2) is a component of normal aqueous humour in many mammalian eyes that increase the ECM production in trabecular meshwork and has been implicated in IOP elevation ${ }^{1}$. TGF- $\beta$ decreases the matrix metalloprotienases(MMP) activity and thus decrease the clearance of ECM. It also decreases the TM cell proliferation and motility.

TGF-B2 also enhance the production of cochlin (ECM protein in TM of POAG eye) that decrease the outflow facility and increase the IOP in monkey and pig organ cultured anterior segment.

Reduction in hyaluronic acid, increase in fibronectin and thrombospondin contribute to the change in the $\mathrm{ECM}^{10}$.Imbalances in response to age related stresses such as oxidative damage to long lived molecule, protein crosslinking and loss of elasticity could trigger excess production of TGFB,IL-1,CD44S, which in turn increases the fibronectin, transform TM cells to myoepithelial cells and decrease ECM breakdown by inhibiting $\mathrm{MMP}^{10}$.There are studies which shows age related decrease in total outflow facility in both monkeys ${ }^{12}$ and humans ${ }^{2}$.

The washout associated increase in outflow in non human species correlates with the extent of physical separation of juxtacanalicular tissue of TM and the inner wall endothelium lining of schlemm's canal, which is deficient in human species ${ }^{1}$.

In rhesus monkeys trabeculum ciliare connecting the uveal portion of TM with the iris root and thereby covering the ciliary muscles $(\mathrm{CM})$ tip $^{11}$. This trabeculum ciliare consists of collagen and elastic fibers that are continuous with the connective tissues of $\mathrm{CM}$ bundle. With advancing age the fibrillar material here increases and the elastic fibers attain a homogenous electron light sheath and thus the total thickness of the fiber increases to lmicrometer ${ }^{11}$.

The autonomic nervous system is thought to be involved in IOP regulation. It has been repeatedly proven that water intake accelerates parasympathetic activation ${ }^{11,13}$.

The parasympathetic activity increased 15 minutes post-WDT and returned to normal at 30 minutes post-WDT. The IOP changed synchronously in the first 15 minutes post WDT and returned to baseline by 60 minutes post WDT. This spike in IOP could be explained by the collapse of schlemm's canal due to parasympathetic over activity ${ }^{16}$.

In humans, reduced formation of giant vacuoles in the inner wall endothelium of schlemm's canal also been proposed for the age related resistance to the outflow(Roy $S$, Boldea $R$, Leuba S, Mermoud A,ARVO Abstract 733,2001).

|www.worldwidejournals.com |

\begin{tabular}{|c|c|c|c|}
\hline AGE & BASELINE IOP & IOP AT 1HOUR & IOP AT 2HOUR \\
\hline $20-30$ & 11.27 & 13.46 & 11.50 \\
\hline $30-40$ & 11.42 & 14.26 & 11.96 \\
\hline $40-50$ & 13.20 & 16.14 & 13.48 \\
\hline $50-60$ & 13.75 & 17.5 & 14.18 \\
\hline
\end{tabular}

INTRAOCULAR PRESSURE (mmHg)

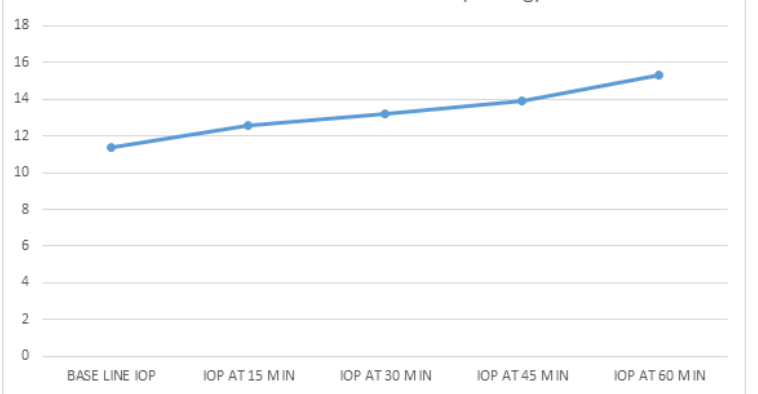

IOP AFTER 1HOUR IN DIFFERENT AGE GROUPS

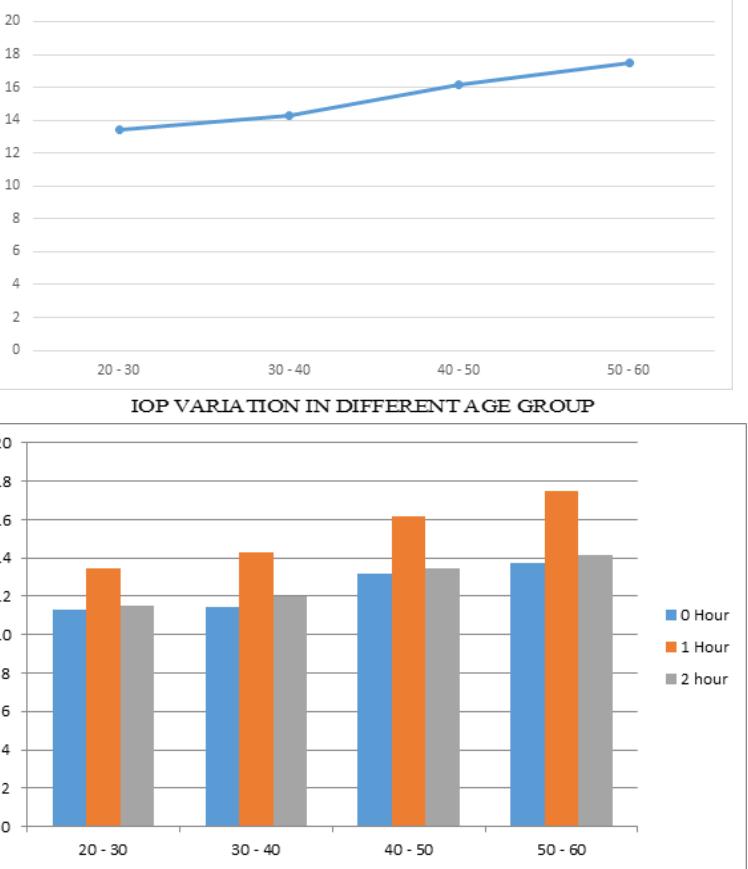

\section{CONCLUSION:}

The above study reveals that as the age increases the effect of drinking water in large quantities within a short period of time causes a rise of IOP which can cause damage to the optic nerve. Hence, the elderly must avoid drinking large quantities of water in a short time, instead take small quantities in intervals in order to prevent the damage to the optic nerve, which may form the basis of low tension glaucoma or normal tension glaucoma.

\section{REFERENCES}

1. Levin, L. A., Kaufman, P. L., \& Alm, A. (Eds.). (2011). Adler's Physiology of the Eye.

2. Becker, B. (1958). The decline in aqueous secretion and outflow facility with age. American journal of ophthalmology, 46(5), 731-736.

3. Goldberg, I., Cunha-Vaz,J., Jakobsen, J.E., Nordmann, J.P., Trost, E., Sullivan, E. K., \& International Travoprost Study Group. (2001). Comparison of topical travoprost eye drops given once daily and timolol $0.5 \%$ given twice daily in patients with open-angle glaucoma or ocular hypertension. Journal of glaucoma, 10(5), 414-422.

4. Susanna, R., Hatanaka, M., Vessani, R. M., Pinheiro, A., \& Morita, C. (2006) Correlation of asymmetric glaucomatous visual field damage and waterdrinking test response. Investigative ophthalmology \& visual science, 47(2), 641-644.

5. Susanna, R.,Vessani, R.M., Sakata, L.,Zacarias,L. C., \& Hatanaka, M. (2005). The relation between intraocular pressure peak in the water drinking test and visual field progression in glaucoma. British journal of ophthalmology, 89(10), 1298-1301.

6. Kerr, N. M., \& Danesh-Meyer, H.V. (2010). Understanding the mechanism of the water drinking test: the role of fluid challenge volume in patients with medically controlled primary open angle glaucoma. Clinical \& experimental 
7. ophthalmology, 38(1), 4-9. Acceptance of the Water-Drinking Test as Compared to the Diurnal Intraocular Pressure Profile by Patients with Primary Open-Angle Glaucoma. Investigative Ophthalmology \&Visual Science, 55(13), 124-124.

8. Gordon, M. O., Beiser, J. A., Brandt, J. D., Heuer, D. K., Higginbotham, E. J., Johnson, C. A., ... \& Ocular Hypertension Treatment Study Group. (2002). The Ocular Hypertension Treatment Study: baseline factors that predict the onset of primary open-angle glaucoma. Archives of ophthalmology, 120(6), 714-720.

9. Leske, M. C., Heijl, A., Hussein, M., Bengtsson, B., Hyman, L., \& Komaroff, E. (2003). Factors for glaucoma progression and the effect of treatment: the early manifest glaucoma trial. Archives of ophthalmology, 121(1), 48-56.

10. Kaufman, P. L. (2005). Changes in aqueous humor dynamics with age and glaucoma. Progress in retinal and eye research, 24(5), 612-637.

11. Peçanha, T., Paula-Ribeiro, M., Campana-Rezende, E., Bartels, R., Marins, J. C. B., \& de Lima, J. R. P. (2014). Water intake accelerates parasympathetic reactivation after high-intensity exercise. International journal of sport nutrition and exercise metabolism, 24(5), 489-496.

12. Crawford, K., \& Kaufman, P. L. (1991). Outflow facility and its response to pilocarpine decline in aging rhesus monkeys. Archives of ophthalmology, 109(6), 879-882.

13. Kawano,Y.U.H.E. I., \& Ferrario, C.M. (1984). Neurohormonal characteristics of cardiovascular response due to intraventricular hypertonic NaCl. American Journal of Physiology-Heart and Circulatory Physiology, 247(3), H422-H428.

14. Gaasterland, D., Kupfer, C., Ross, K., \& Gabelnick, H. L. (1973). Studies of aqueous humor dynamics in man: III. Measurements in young normal subjects using norepinephrine and isoproterenol. Investigative subjects using norepinephrine and isoprc
Ophthalmology \&Visual Science, 12(4), 267-279.

15. Chen, W., Chen, L., Chen, Z., Xiang, Y., Liu, S., Zhang, H., \& Wang, J. (2018). Influence of the water-drinking test on intraocular pressure, Schlemm's canal, and autonomic nervous system activity. Investigative ophthalmology \& visual science, 59(8), 3232-3238.

16. Goel, M., Picciani, R. G., Lee, R. K., \& Bhattacharya, S. K. (2010). Aqueous humor dynamics: a review. The open ophthalmology journal, 4,52 . 